\title{
CURAR Y CUSTODIAR. LA CRONICIDAD EN EL MANICOMIO LA CASTAÑEDA, CIUDAD DE MÉXICO, 1910-1968
}

\author{
Cristina Sacristán \\ Instituto Mora, Centro Público Conacyt, México. \\ csacristan@mora.edu.mx \\ ORCID iD: http://orcid.org/0000-0002-9587-7096
}

Recibido: 07 abril 2017; Aceptado: 18 junio 2017.

Cómo citar este artículo/Citation: Sacristán, Cristina (2017), "Curar y custodiar. La cronicidad en el Manicomio La Castañeda, Ciudad de México, 1910-1968", Asclepio 69 (2): p193. doi: http://dx.doi.org/10.3989/asclepio.2017.16

RESUMEN: El artículo cuestiona el binomio que asocia la cronicidad y la incurabilidad de las enfermedades mentales con el custodialismo del manicomio mediante un estudio de caso, el Manicomio La Castañeda de México, 1910-1968. Se contrastan los discursos sobre la cura y la cronicidad que elaboraron los psiquiatras mexicanos y las tendencias estadísticas de los pacientes ingresados: nuevas admisiones, reingresos, altas, duración de la estancia y diagnósticos a la luz de los nuevos tratamientos. Concluye que para los médicos, la función terapéutica del manicomio se vio muy golpeada por la cronicidad y la sobrepoblación, pero según las estadísticas, el $80 \%$ de los pacientes sólo tuvo un ingreso con una internación de 15 meses y las largas estancias de los que reingresaron no impactaron estadísticamente; las dos terceras partes de los enfermos salieron del manicomio, y desde los años cincuenta en el contexto de las nuevas terapéuticas.

PALABRAS CLAVE: Manicomio; Cronicidad; Demografía Psiquiátrica; México.

ABSTRACT: The article questions the binomial that associates the chronicity and incurability of mental illness with the custodialism of the asylum through a case study, Asylum La Castañeda in Mexico, from 1910 to 1968 . We contrast the discourses about the cure and chronicity constructed by Mexican psychiatrists and the statistical trends of patients admitted: new admissions, readmissions, discharges, length of stay, and diagnoses in the light of new treatments. We concluded that according to the doctors, the asylum therapeutic function was severely affected by chronicity and overpopulation, but according to statistics, $80 \%$ of the patients had only one admission with a 15-month hospitalization and the long-term confinement rates of readmissions did not impact statistically; twothirds of the patients left the asylum, and since the 1950s in the context of new therapeutics.

KEY WORDS: Mental Hospital; Chronicity; Psychiatric Demography; Mexico. 
No sé por qué en lo que se refiere a la patología del sistema nervioso está tan exagerado el concepto de incurabilidad. Juan Peón del Valle ${ }^{1}$

Hace ya varias décadas, reflexionando sobre la psiquiatría contemporánea, Lantéri-Laura mostró el momento en que la noción de cronicidad se convirtió en una dimensión fundamental del pensamiento psiquiátrico francés. Mientras que durante la primera mitad del siglo XIX los alienistas creyeron en la curación de la locura y la concibieron como un estado, "un paréntesis en la existencia", que podía ser tratada bajo un régimen de aislamiento, la cronicidad no ocupó un lugar importante. Por el contrario, en el último tercio de esa centuria se fue imponiendo la idea de que algunas patologías mentales evolucionaban hacia la cronicidad de manera irreversible. Así, nociones como el delirio crónico, la demencia o la teoría de la degeneración desvanecieron el optimismo que había caracterizado a las dos primeras generaciones de alienistas sobre las posibilidades de curación de los enfermos y el regreso a su vida normal, (Lantéri-Laura, 1972, pp. 552-557).

Posteriormente, la historiografía abundó sobre la manera en que el degeneracionismo apuntaló la concepción orgánica de la enfermedad mental al anclar su etiología en la predisposición hereditaria y la lesión cerebral y con ello, la noción de incurabilidad de algunos padecimientos. Este desarrollo teórico tuvo lugar de manera paralela al aumento de la población internada en los manicomios, por lo que contribuyó aún más a sembrar el pesimismo. Todo ello resultó en un cuestionamiento sobre el valor científico de la psiquiatría, aunque dio pie para que ésta ampliara su intervención más allá del asilo (Huertas, 1987, pp. 1732; Álvarez, Huertas y Peset, 1993; Caponi, 2012, pp. 81-98). La historiografía también se interesó por la relación entre los confinamientos prolongados de los pacientes en los manicomios y que muchos de ellos fueran considerados aptos para el trabajo dentro de la institución. Si bien la productividad pudo valorarse como un signo de recuperación, también incentivó la existencia de "crónicos" para la sobrevivencia de los establecimientos (Castel, 1980, pp. 264-265; Vezzetti, 1985, pp. 66-77; Comelles, 1997; Mills, 1999; Eraso, 2002; Ernst, 2016), problemática que el mismo Lantéri-Laura adelantó al señalar que en estos internamientos las razones terapéuticas pasaban a un segundo plano frente a la rentabilidad que suponía una mano de obra abundante y barata (Lantéri-Laura, 1972, pp. 565-568). Por otro lado, también se ha estudiado cómo ante el crecimiento de los pacientes considerados crónicos, el debate sobre la reforma del asilo se volvió inevitable, ya que el propio encierro fue vis- to como generador de cronicidad y, tanto en Francia como en otros países, se abrió la discusión sobre la necesidad de ofrecer alternativas que hicieran menos severo el aislamiento, como los servicios de puertas abiertas. Se planteó el imperativo de desdoblar la atención en distintos niveles: hospitales psiquiátricos para enfermos considerados curables, asilos-colonia para crónicos o curables a muy largo plazo, y atención ambulatoria en dispensario o consultorio para quienes no requerían ser hospitalizados, pese a que el manicomio continuó siendo el modelo más extendido (Dowbiggin, 1991, pp. 93-110; Campos, 2001; Huertas, 2002, pp. 174-208). Pero quizás haya sido la correspondencia entre cronicidad/incurabilidad de la enfermedad mental y custodialismo de los manicomios la perspectiva que arraigó con más fuerza en la historiografía, ya que las estancias largas habrían provocado la sobrepoblación de los asilos e impedido brindar una atención adecuada por la inevitable masificación, derivando en instituciones que tendieron al confinamiento más que al tratamiento, un escenario no previsto en el plan inicial cuando el tratamiento moral prometió la cura para establecimientos de cien o doscientos pacientes. Esta interpretación se ha sostenido a partir del crecimiento en el número de internos, pero también por los desesperados testimonios de los médicos que vieron cómo los crónicos abortaban la esperanza puesta en el manicomio. ${ }^{2}$

El objetivo de este trabajo es situarnos en esta última problemática para cuestionar el binomio que asocia la cronicidad y la incurabilidad con el custodialismo y para ello, disponemos ahora de una novedosa herramienta que permite conocer el perfil y la dinámica de las poblaciones que nutrieron los manicomios, la demografía psiquiátrica. ${ }^{3}$ Nuestro objeto de estudio, el Manicomio La Castañeda de México, institución pública situada en la capital del país, atendió a 61,480 pacientes desde su apertura en 1910 hasta su cierre y demolición en 1968, cuando en México se pretendió poner fin al modelo asilar. Durante este lapso de tiempo, el número de enfermos casi triplicó el cupo original y obligó a ampliaciones sucesivas siempre insuficientes, por lo que padeció de sobrepoblación y hacinamiento. ${ }^{4}$ Investigaciones recientes sobre este manicomio han revelado indicadores demográficos que dan cuenta de una gran movilidad entre la población recluida (Ríos Molina et al., 2016), hallazgo que contrasta con los conocidos discursos de los facultativos que lo dirigieron, cuyo entusiasmo inicial se fue apagando con el tiempo. Es por ello que metodológicamente nos interesa confrontar las fuentes discursivas con los registros cuantitativos. Así, desde un doble 
acercamiento, nos proponemos cuestionar la idea de que el manicomio haya sido una institución meramente custodial, ya que su carácter asilar, que sin duda lo tuvo, pudo coexistir con su función terapéutica. Este planteamiento ya fue trazado hace algunos años al calor de la historiografía del control social cuando se buscaba redimensionar el peso de la familia frente al del Estado y la psiquiatría (McGovern, 1986). En nuestro caso, la familia jugó un papel muy importante en las altas de los pacientes, pero también los médicos y los propios internos, como enseguida se verá.

\section{LOS DICHOS DE LOS MÉDICOS}

Son muchos los testimonios que a lo largo de seis décadas dieron cuenta del estado del manicomio y de sus necesidades: artículos en revistas especializadas, informes administrativos dirigidos a las autoridades y entrevistas en los periódicos en respuesta a denuncias publicadas, nutren este corpus documental. De todos ellos, hemos seleccionado los que consideramos más representativos de la postura asumida por los facultativos que dirigieron la institución, pero también la de quienes desde fuera se implicaron en ella. También hemos construido una periodización que nos permitirá analizar los indicadores demográficos escogidos para evaluar el carácter custodial y/o terapéutico de La Castañeda.

Pese a que durante su primera década (1910-1920), el manicomio se vio seriamente afectado por las condiciones de guerra que vivió el país (Ríos Molina, 2009), el optimismo sobre las perspectivas de curación de los enfermos mentales era bastante notable. Inaugurado en la antesala de la revolución mexicana, se planeó para 1,300 camas con posibilidad de extenderlas a 2,000 (Valdés Fernández, 1995, pp. 135-144), y en sus primeros años el descenso en el número de ingresos fue la nota dominante a causa de la inestabilidad política y la desorganización administrativa provocada por la contienda bélica que afectó a la capital en coyunturas muy violentas (Ríos Molina, et al., 2016, p. 4). El descrédito de la institución se manifestó plenamente en la siguiente década, pero se presagiaba desde ésta. Por ello, cuando en 1919 el doctor Agustín Torres, director en ese momento, recibió una petición de la Procuraduría General de la República, la máxima autoridad en materia de justicia, para que entregara la lista de todos los internos que no hubieran sido declarados incapaces con el fin de promover su interdicción legal, la negativa no se hizo esperar. Torres explicó que una medida de ese tipo sembraría la desconfianza entre las familias haciéndolas desistir de llevar a sus enfermos, pero además señaló que los padecimientos mentales eran muy diversos y su perspectiva de curación también: algunos enfermos pasaban por estados transitorios; otros cuyo mal se consideraba "fatalmente irremediable", volvían en uno o dos meses "al seno de la sociedad"; incluso enfermos ingresados "en pleno delirio, con gran excitación" podían ser tratados con buenos resultados, y hasta en padecimientos irreversibles y progresivos como la parálisis general, los tratamientos (no especificó cuáles) permitían "a los enfermos salir y desempeñar trabajos remunerados". ${ }^{5}$ Aunque el contexto -someter a un procedimiento judicial a los pacientes- obligaba a reaccionar legitimando a la psiquiatría, la creencia en la curación de las enfermedades mentales es de destacarse.

En la siguiente década (1921-1931), la institución comenzó a recuperarse de la guerra, hecho que se manifestó en el incremento de nuevos ingresos, pero a un ritmo tolerable en relación con el cupo máximo permitido, por lo que aún no se prendían las alarmas ante el aumento de pacientes, aunque sí respecto a las instalaciones y la atención recibida (Ríos Molina, et al., 2016, p. 4). El optimismo terapéutico continuó, pero confrontado con el que podemos considerar el primer testimonio absolutamente demoledor sobre el carácter custodial de La Castañeda, que data de 1925. En ese momento, la Beneficencia Pública de la que dependía el manicomio, comisionó al médico Enrique 0. Aragón para que presentara un informe sobre las necesidades de la institución. Años atrás, Aragón había presidido la sección de neurología y psiquiatría de la Academia Nacional de Medicina y, con estas credenciales, señaló varios hechos que le parecieron graves: gran número de internos sin diagnóstico, historias clínicas muy deficientes, personal médico sin preparación y total ausencia de investigación, pero en ningún momento atribuyó la decadencia existente al excesivo número de crónicos ni a las carencias de la psiquiatría como ciencia, sino al hecho de que ésta no se practicara, dejando a los pacientes en el más absoluto abandono (Aragón, 1943, pp. 417-436).

Comenzó entonces un proceso de reforma con la llegada a La Castañeda de un joven director, Manuel Guevara Oropesa, que lo sería de 1932 a 1944, salvo los años de 1934 a 1938. Constituye el tercer momento de nuestra periodización, etapa caracterizada por el mayor crecimiento registrado en los ingresos de pacientes hasta alcanzar un máximo histórico de 1,663 en 1944 frente al mínimo de 384 que tuvo lugar en 1915 y a la tendencia a la estabilización que vendría después de 1945 (Ríos Molina, et al., 2016, pp. 4-5). La ocupación 
total del manicomio para 1943 fue de 3,414 pacientes, ${ }^{6}$ número elevadísimo si pensamos en la capacidad inicial de 1,300 camas. Durante estos años, Guevara Oropesa, que también fundó la Sociedad Mexicana de Neurología y Psiquiatría en 1937, echó a andar varias estrategias para hacer frente a la sobrepoblación y a la maltrecha infraestructura: dio continuidad a la terapia ocupacional, que había iniciado su antecesor en el cargo; estableció un periodo de observación de 72 horas en el proceso de admisión; creó el llamado Pabellón Central, una unidad destinada a los agudos enfocada en las terapias de choque, quirúrgicas y posteriormente farmacológicas, donde reunió los medios de diagnóstico y tratamiento que la institución muy lentamente pudo ir adquiriendo (rayos $X$, electroencefalógrafo, gabinete de electropirexia y salas para el tratamiento con malarioterapia e insulinoterapia, equipo de electrochoques, laboratorio de psicometría y psicodiagnóstico, departamento de neurocirugía); finalmente, abrió el primer consultorio fuera del manicomio para tratar a los enfermos en consulta externa. A todo ello, sumó una remodelación física de las instalaciones gracias a presupuestos extraordinarios que fueron autorizados por el gobierno federal (Sacristán, 2001, 2005, 2017; Valverde Pérez, 2016). Si bien los años que van de 1932 a 1944 fueron muy complejos para la institución por la sobrepoblación, culminaron en 1944 con la aprobación del primer proyecto para remitir 400 pacientes crónicos fuera del manicomio, una Granja ubicada en el centro del país, lo que pudo ser percibido como un indicio de cambio. ${ }^{7}$

Este proyecto fue elaborado por Guevara Oropesa como parte de un Programa de Asistencia Psiquiátrica donde consideró también la creación de un nuevo manicomio, que nunca se construyó. Dado que la Granja se ideó precisamente para desahogar a La Castañeda de los pacientes crónicos, el director contempló una definición general de cronicidad para seleccionar a los que serían trasladados, los "diagnosticados como incurables" o en los que hubieran "fracasado" todos los tratamientos, y los dividió en tres grupos por cuestiones prácticas: a) aquellos con "un padecimiento crónico incurable", pero "compatible con una buena salud física" para trabajar, esto es, "la mayor parte de los esquizofrénicos, los imbéciles y los débiles mentales"; b) aquellos cuyos "padecimientos son crónicos, incurables", que "requieren un tratamiento continuado y que aun cuando mejoran por temporadas no pueden volver al medio social ni familiar" como los epilépticos esenciales, y c) "los enfermos crónicos incurables que sólo requieren asilo" por haber "sido sometidos a todos los tratamientos que puedan mejorarlos", pero sin resultado alguno. Esta clasificación consideraba a los crónicos trabajadores, a los crónicos con mejorías intermitentes y a los crónicos definitivamente incurables. Pese a este panorama, Guevara Oropesa veía el futuro con cierto optimismo, pues en su opinión "afortunadamente para los enfermos mentales el pronóstico de muchos de sus padecimientos se ha modificado favorablemente por la aplicación oportuna y eficiente de tratamiento apropiado". ${ }^{8}$

Ese mismo año de 1944, la doctora Mathilde Rodríguez Cabo, psiquiatra y miembro del Consejo Psiquiátrico de Toxicomanías e Higiene Mental, uno de los pocos espacios de confluencia y debate entre la psiquiatría y el Estado mexicano donde los médicos presentaban a las autoridades los problemas que les aquejaban (Ríos Molina, 2016, pp. 68-80), ponía el dedo en la llaga cuando, con motivo de la presentación de su propuesta para crear un programa de servicio social, señaló que durante muchos años La Castañeda "se limitó a ser un almacén de enfermos mentales, con reducidas o nulas posibilidades de acción terapéutica, preventiva y de higiene social frente a los asilados, a sus familiares y a la comunidad". Atribuía esta delicada situación al hecho de que al momento del ingreso se perdía el vínculo con la familia y el médico se veía obligado a "aceptar la permanencia indefinida de algunos enfermos dentro de la institución, aún a sabiendas de que la reintegración al medio social, familiar o no familiar, podía realizarse si se llenaba el requisito de una vigilancia y una acción social continuadas". Tampoco se mantenía dicho contacto tras el alta, de donde se seguían las recaídas sin que el manicomio pudiera intervenir a tiempo. ${ }^{9}$ Para Rodríguez Cabo la institución era un "almacén" o depósito de enfermos, entre otras razones, por el poco interés de las familias que abandonaban a sus pacientes, lo que no fue cierto en todos los casos, como se verá.

Durante el periodo que va de 1945 a 1959 las admisiones se estabilizaron, posiblemente por la apertura de la consulta externa en el periodo anterior, frenando la tendencia ascendente aunque en niveles bastante altos, de entre 1,200 y 1,600 ingresos cada año (Ríos Molina, et al., 2016, p. 4). A ello se aunó la promesa incumplida por parte del gobierno federal de abrir más Granjas para crónicos en distintos puntos del país, ya que las dos siguientes esperaron trece años para su apertura en 1958. En este mismo año, Manuel Velasco Suárez tomó posesión como titular de la Dirección de Neurología, Salud Mental y Rehabilitación de la Secretaría de Salubridad y Asistencia. Su arribo a este cargo provocó un cambio en las políticas de salud mental en 
el país de cara a un posible cierre de La Castañeda para dar paso a la psiquiatría comunitaria, en consonancia con las tendencias internacionales (Velasco Suárez, 1964; Secretaría, 1964, pp. 153-155).

A partir del arribo de Velasco Suárez como titular responsable de la salud mental del país, se instauró un mecanismo unilateral y vertical en la toma de decisiones, desde las altas esferas del Estado, sin involucrar a la comunidad de psiquiatras (Hernández Lara, 2014, p. 68), dinámica que determinó la última etapa del manicomio (1960-1968). El traslado de los pacientes y la apertura de nuevas instituciones se acompañó de una exposición mediática que lo desprestigió aún más, generando un clima propicio para justificar su cierre y demolición (Sacristán, 2011). De hecho, el juicio más duro sobre la institución lo encontramos hacia el final de sus días. En 1960, cuando La Castañeda albergaba 3,217 internos, dos médicos desempolvaron los archivos del establecimiento con motivo de su 50 Aniversario para recorrer su historia. Su director José Luis Patiño, que había entrado al manicomio en 1936 y el psiquiatra Ignacio Sierra Macedo, que lo hizo en $1942,{ }^{10}$ dejaron claro que "la lucha fundamental de los médicos y directores ha estado dirigida a mantener al Manicomio General como un Hospital Psiquiátrico", es decir, a no olvidar su condición terapéutica pese a la "sobrepoblación obligada", que atribuyeron a tres causas:

1a.- La imperiosa necesidad de no rechazar jamás a ningún enfermo mental que amerite internamiento. 2a.- La circunstancia de que absorbe a todos los pacientes con bajos recursos del Distrito Federal y a la gran mayoría del resto del país. 3a.- La prolongada estancia que requieren para su atención este tipo de enfermos y el elevado porcentaje de crónicos e incurables. Este último hecho ha sido y es uno de los obstáculos más graves con que ha tropezado la institución.

Entre las conclusiones de su estudio, los médicos Patiño y Sierra señalaron la urgencia de encontrar "soluciones que permitan descongestionar el sobrecupo de la institución para elevar su nivel científico y social". ${ }^{11}$

Este interesante documento, elaborado por médicos muy experimentados y conocedores del manicomio, plantea que el nudo gordiano fue la sobrepoblación, provocada por las largas estancias de los pacientes incurables. Sin embargo, alerta sobre dos elementos muy importantes: dada su condición de institución pública, no podía rechazar ningún ingreso, y su radio de acción iba más allá de la capital, pues recibía enfermos de todo el país. En resumen, el manicomio debió hacer frente al exceso de demanda y a cuidados psiquiátricos prolongados.
En este recuento de testimonios puede observarse que la atención médica fue la prioridad de quienes dirigieron La Castañeda, y conforme pasaba el tiempo, la solución al problema de los crónicos y al hacinamiento se fue volviendo cada vez más lejana. Veamos qué nos dicen las estadísticas.

\section{LAS RAZONES DE LOS NÚMEROS}

Las variables que utilizaremos para valorar desde la demografía psiquiátrica el carácter custodial y/o terapéutico de La Castañeda serán: 1) la relación entre los ingresos y los egresos, 2) la relación entre los ingresos y los reingresos, 3) el tiempo de permanencia de los pacientes en el manicomio, 4) las altas por indicación médica, por petición de la familia y por intervención del propio paciente, y 5) los diagnósticos en relación con los tratamientos. Para acercarnos a la población de La Castañeda con estos indicadores, contamos con una base de datos elaborada a partir de una muestra aleatoria del $20 \%$ del total de la población que ingresó entre 1910 y 1968, que fue de 61,480 pacientes. Una vez que se eliminaron los registros sin diagnóstico (257 casos), los que recibieron más de un diagnóstico al momento del primer ingreso (1,361 casos) y aquellos cuyo diagnóstico no fue una enfermedad psiquiátrica (42 casos), la muestra se redujo de 12,296 registros a 10,641 , por lo que nuestra muestra para esta investigación conserva el $86.5 \%$ de la muestra original. Por otro lado, hay que aclarar que la información relativa a los ingresos se obtuvo de los libros de admisión del manicomio y la de los reingresos de los expedientes clínicos. ${ }^{12}$

Frente al discurso de los médicos sobre el problema de la cronicidad y la sobrepoblación que acabamos de ver, analizaremos un primer dato, la relación entre ingresos y egresos. En la figura 1 se observa que los ingresos casi siempre superaron a los egresos salvo en tres momentos muy concretos que no modificaron la tendencia general. El primero de ellos corresponde a los años 1914-1915, los más duros en relación con el conflicto bélico, que provocaron hambrunas y epidemias en la ciudad y con una afectación importante en el manicomio (Ríos Molina, et al., 2016, p. 4). Estos egresos se vieron influidos por la alta mortandad, ya que mientras para el periodo 1910-1968, la mortalidad en La Castañeda fue de $24.2 \%$, durante los años $1910-1920$ se elevó a $39.52 \%$, como puede verse en la tabla 1. El segundo momento donde los egresos superaron a los ingresos fueron los años posteriores a 1945, fecha de apertura de la primera Granja para enfermos crónicos, hecho que no podemos atribuir a 
la mortalidad, ya que ésta descendió a $20.03 \%$, sino a los traslados de pacientes crónicos. Finalmente, durante los años que van de 1963 a 1968, previos al cierre de La Castañeda, los egresos también estuvieron por encima de los ingresos a causa del proceso que anticipaba el desmantelamiento de La Castañeda; en este caso las altas se incrementaron por la reubicación de pacientes.

El análisis de esta variable muestra una correspondencia entre los ingresos y los egresos, como si los médicos hubieran tenido la precaución de mantener un equilibrio entre unos y otros, ya que las trayectorias de ambas curvas están bastante cercanas. Analizaremos ahora la relación entre ingresos y reingresos.

Del total de pacientes del manicomio, ocho de cada diez ingresaron sólo una vez (79.63\%) obteniendo un alta definitiva. El $20.37 \%$ restante se repartió en trece reingresos, concentrándose la mayoría de los pacientes en el segundo ingreso (trece pacientes de cada cien) y en el tercero (cuatro pacientes de cada cien), ya que a partir del cuarto ingreso, el número de pacientes disminuyó a uno de cada cien o menos (tabla 2).

Figura 1. Ingresos y egresos en el Manicomio La Castañeda, 1910-1968 ( $n=10,641)$

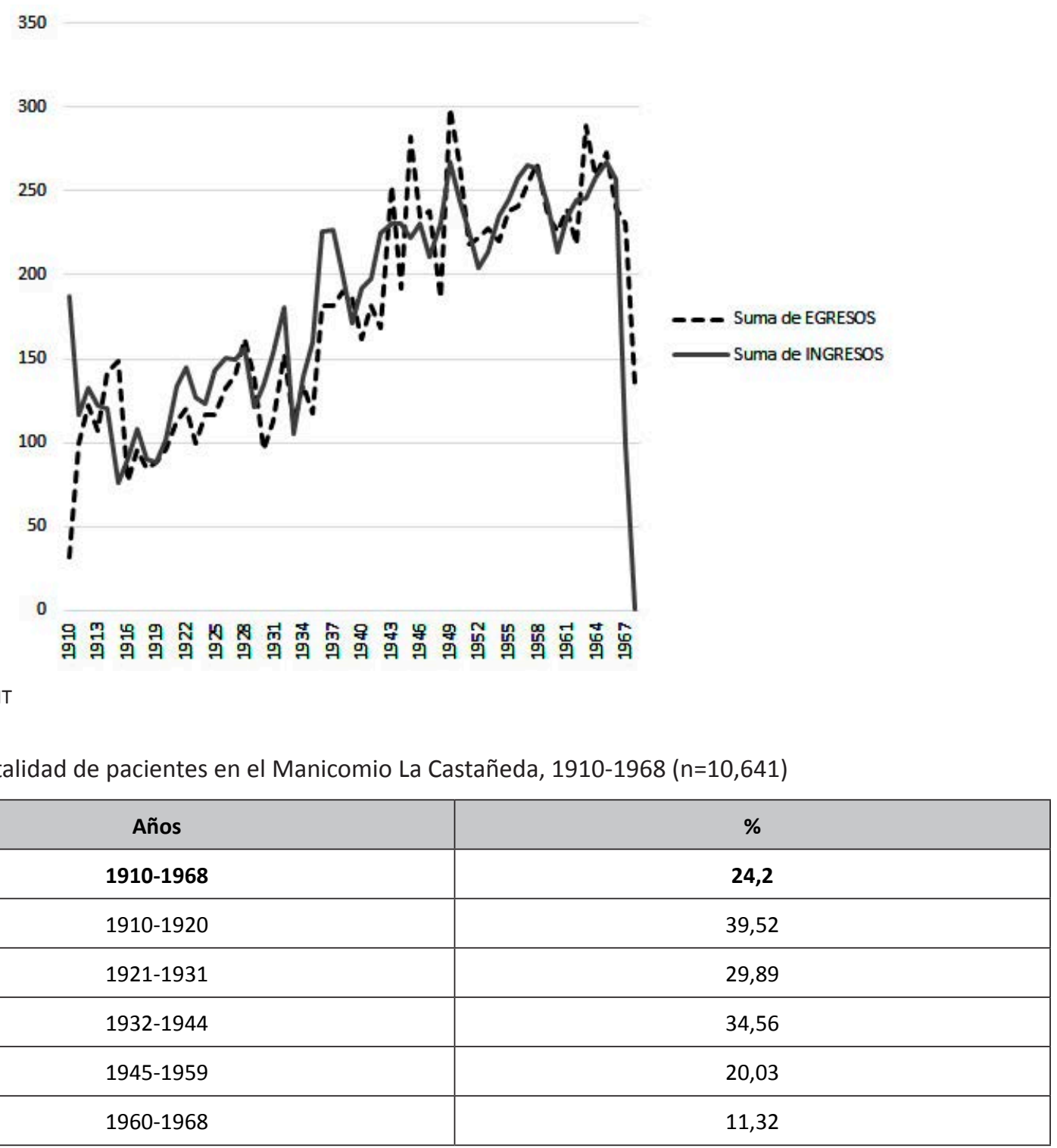

Fuente: BD-PAPIIT 
Tabla 2. Pacientes que ingresaron y reingresaron al Manicomio La Castañeda, $1910-1968$ ( $n=10,641)$

\begin{tabular}{|c|c|c|c|c|c|c|c|c|c|c|c|c|c|c|}
\hline Ingresos & 1 & 2 & 3 & 4 & 5 & 6 & 7 & 8 & 9 & 10 & 11 & 12 & 13 & 14 \\
\hline$\%$ & 79,63 & 13,33 & 4,14 & 1,25 & 0,76 & 0,36 & 0,23 & 0,056 & 0,046 & 0,056 & 0,037 & 0,009 & 0,037 & 0,009 \\
\hline$n=$ & 8.474 & 1.419 & 441 & 134 & 81 & 39 & 25 & 6 & 5 & 6 & 4 & 1 & 4 & 2 \\
\hline
\end{tabular}

Fuente: BD-PAPIIT

Suponiendo que todos los reingresos hayan obedecido a recaídas en la enfermedad, tendríamos que la quinta parte de los internos eventualmente podría cronificarse. Con el fin de avanzar sobre esta posibilidad, compararemos cuánto tiempo permanecieron en el manicomio los que sólo tuvieron un ingreso y los que reingresaron, considerando en este caso hasta el tercer ingreso (en conjunto representan el $97.1 \%$ de nuestra muestra). Si bien los que sólo tuvieron una estancia también pudieron cronificarse y morir en el manicomio, la causa de muerte pudo deberse a su estado general de salud o a las condiciones sanitarias de la institución y no necesariamente a su enfermedad, de ahí que sólo tomemos a esa quinta parte de los internos como la que tendría más tendencia a la cronicidad.

Quienes sólo ingresaron una vez a La Castañeda tuvieron un promedio de estancia de un año y tres meses, como puede verse en la tabla 3 . Este promedio fue en ascenso hasta alcanzar el punto más alto en los años que van de 1932 a 1944 llegando a un año y ocho meses, decreciendo a partir de entonces de ma- nera muy importante a poco más de 7 meses para la última década, de 1960 a 1968. El periodo 1932-1944 no sólo acumuló el promedio de internación más alto, sino también los máximos históricos en el ingreso de nuevos pacientes, como ya se dijo.

La situación es muy diferente entre los pacientes que tras un primer ingreso, salieron de la institución y reingresaron (tabla 4). En este caso, la primera entrada al manicomio tuvo una estancia de tan sólo nueve meses y medio, llegando entre los años de 1932 a 1944 a quince meses y disminuyendo de manera drástica en los últimos años a menos de cinco meses. Sin embargo, en el segundo ingreso a la institución, el tiempo de estancia aumentó de manera espectacular, ya que se multiplicó por cinco, pues pasó a tres años y nueve meses para el periodo 1910-1968, llegando a cinco años y cuatro meses en el periodo más crítico, de 1932 a 1944, y disminuyendo a dos años entre 1960 a 1968. En el tercer ingreso, el tiempo de internamiento aumentó aún más a cuatro años y medio, pero disminuyó durante los años intermedios (1921-1944) para

Tabla 3. Duración de la estancia en pacientes con un solo ingreso en el Manicomio La Castañeda, 1910-1968

\begin{tabular}{|c|c|}
\hline \multirow{3}{*}{ Años } & Ingreso único \\
\hline & $79.63 \%(n=8,474)$ \\
\hline & Promedio en meses \\
\hline $1910-1968$ & 14,9 \\
\hline $1910-1920$ & 16,6 \\
\hline 1921-1931 & 17,3 \\
\hline $1932-1944$ & 20,4 \\
\hline $1945-1959$ & 13,2 \\
\hline $1960-1968$ & 7,3 \\
\hline
\end{tabular}

Fuente: BD-PAPIIT 
Tabla 4. Duración de la estancia en pacientes que reingresan (primer, segundo y tercer ingreso) en el Manicomio La Castañeda, 1910-1968

\begin{tabular}{|c|c|c|c|}
\hline \multirow{2}{*}{ Años } & Primer ingreso & Segundo ingreso & Tercer ingreso \\
\cline { 2 - 4 } & $20.37 \%(n=2,167)$ & $13.33 \%(n=1,419)$ & \multirow{2}{*}{$4.14 \%(n=441)$} \\
\cline { 2 - 4 } & \multicolumn{3}{|c|}{ Promedio en meses } \\
\hline $1910-1968$ & $\mathbf{9 , 4}$ & $\mathbf{4 5 , 4}$ & $\mathbf{5 4 , 1}$ \\
\hline $1910-1920$ & 10,4 & 50,5 & 61,2 \\
\hline $1921-1931$ & 8,2 & 63,6 & 61,1 \\
\hline $1932-1944$ & 15,1 & 64,8 & 75,1 \\
\hline $1945-1959$ & 8,2 & 48,9 & 31,8 \\
\hline $1960-1968$ & 4,8 & 24,6 & 33,8 \\
\hline
\end{tabular}

Fuente: BD-PAPIIT

crecer todavía más en las últimas décadas (19451968). Quienes tuvieron dos ingresos (13.33\% de la muestra) acumularon un promedio de cuatro años y medio para el periodo 1910-1968 y de seis años y ocho meses entre 1932 y 1944, aunque disminuyeron a dos años y medio para 1960-1968. Quienes tuvieron tres ingresos ( $4.14 \%$ de la muestra) acumularon nueve años para el periodo 1910-1968, casi once años para las décadas intermedias y poco más de cinco años para el último periodo.

Cabe preguntarse qué tanto impactaron estos confinamientos tan prolongados en las tendencias de la institución. En la tabla 5, donde hemos reunido a todos los pacientes, se puede apreciar que el promedio de estancia para todo el manicomio fue de 13.5 meses, por lo que quienes reingresaron, pese a una larga reclusión, no lograron modificar el movimiento general que guarda una gran similitud con los que únicamente ingresaron una vez (tabla 3). Tras este análisis, creemos que la dinámica respecto a la duración del internamiento estuvo dominada por el $80 \%$ de los pacientes que con un solo ingreso de 15 meses de estancia obtuvo el alta definitiva (30.31\% de ellos murió en el manicomio) (figura 4).

Aunque no podemos asegurar que todos los pacientes que reingresaron se cronificaron, pues tendríamos que analizar si al reingresar entraron con el mismo diagnóstico de un inicio, y acudir para ello a los expedientes clínicos, además de conocer cuánto tiempo transcurrió entre un ingreso y otro (el paciente pudo estar bien durante un lapso de tiempo importante), ${ }^{13}$ estas largas estancias seguramente influyeron en el ánimo de los psiquiatras que veían con preocupación cómo la vocación terapéutica del manicomio fracasaba al menos, con un sector de los enfermos. Sin embargo, la frustración que se advierte en los médicos Patiño y Sierra, tras medio siglo de existencia de La Castañeda, requiere que sigamos buscando una explicación ya que para 1960 , si bien las admisiones se mantenían en cifras muy elevadas, (entre 1,400 y 1,600 cada año) (Ríos Molina, et al., 2016 , p. 4), el promedio de estancia que se avizoraba en el horizonte era, lo sabemos ahora, de menos de siete meses (tabla 5). Pasaremos entonces al análisis de las altas para ver si guardan alguna relación con los señalamientos de los médicos sobre la sobrepoblación y la cronicidad.

Tabla 5. Duración de la estancia en ingresos y reingresos en el Manicomio La Castañeda, 1910-1968 $(n=10,641)$

\begin{tabular}{|c|c|}
\hline Años & Promedio en meses \\
\hline $\mathbf{1 9 1 0 - 1 9 6 8}$ & $\mathbf{1 3 , 5}$ \\
\hline $1910-1920$ & 15 \\
\hline $1921-1931$ & 14,7 \\
\hline $1932-1944$ & 19,3 \\
\hline $1945-1959$ & 11,9 \\
\hline $1960-1968$ & 6,8 \\
\hline
\end{tabular}

Fuente: BD-PAPIIT 
Para todo el manicomio, la causa más importante de egreso entre ingresos y reingresos, fue la solicitud de la familia (29.38\%), por encima de la mortalidad (24.20\%), ocupando un tercer lugar la remisión de la enfermedad (13.08\%) y no regresar del permiso que se concedía cuando el médico así lo consideraba (12.32\%). Si el paciente no volvía una vez transcurrido el tiempo autorizado, la institución lo registraba como un alta y para aceptar su regreso aplicaba el procedimiento normal de admisión, de ahí que lo estemos tomando con un egreso. ${ }^{14}$ La fuga como causa de salida (5.66\%) estuvo por encima de la mejoría (3.99\%) y de la curación (2.65\%) (figura 2).

Con el fin de profundizar en los factores que motivan la salida, vamos a agrupar estos criterios utilizados por los médicos, en tres categorías:

- Altas por indicación médica: aquellas que se dieron por decisión del facultativo y que corresponden a las altas por curación, por mejoría, por remisión, por ser apto para vivir en familia y por llegar al término del tratamiento.

- $\quad$ Altas por petición de la familia: las que obedecieron a la solicitud de la familia o del responsable, ya que los enfermos podían ser dados de alta por solicitud de la familia estuvieran aliviados o no; en estos casos el parecer de los parientes podía imponerse sobre el del médico, salvo cuando el sujeto fuera peligroso. ${ }^{15}$
- Altas por intervención del paciente: las que se debieron a la solicitud del propio paciente (los pacientes podían salir solos si eran mayores de edad y no habían entrado bajo la calidad de reos), a las fugas y por no regresar del permiso. En este último caso, si bien los permisos eran concedidos por el médico y la familia pudo intervenir para no volver con el paciente, también podía decidirlo el enfermo, incluso fugarse del seno familiar para evitar el encierro, de ahí que lo hayamos puesto en este rubro. ${ }^{16}$

Bajo estas categorías (tabla 6), puede advertirse que las dos terceras partes de los pacientes salieron del manicomio. ${ }^{17}$ Pudieron hacerlo curados o sin ninguna mejoría, como agudos o como crónicos, con posibilidad de recuperar el lazo social o para permanecer bajo los cuidados de la familia, o incluso pudieron escapar con un destino incierto. Todas estas posibilidades tienen algo en común, quizá no tanto de cara al sujeto, pero sí para la institución: los pacientes no se perpetuaron en ella aliviando el peso que su estadía significaba en términos de la capacidad del establecimiento para proporcionarles alojamiento, alimentación, vestido, cuidados higiénicos y tratamiento, todo lo cual requería de un presupuesto que no siempre era proporcionado por el Estado y que, sin duda, angustiaba a los médicos. ${ }^{18}$

Figura 2. Condición de salida de pacientes en ingresos y reingresos en el Manicomio La Castañeda, 1910-1968 (n=10,641)

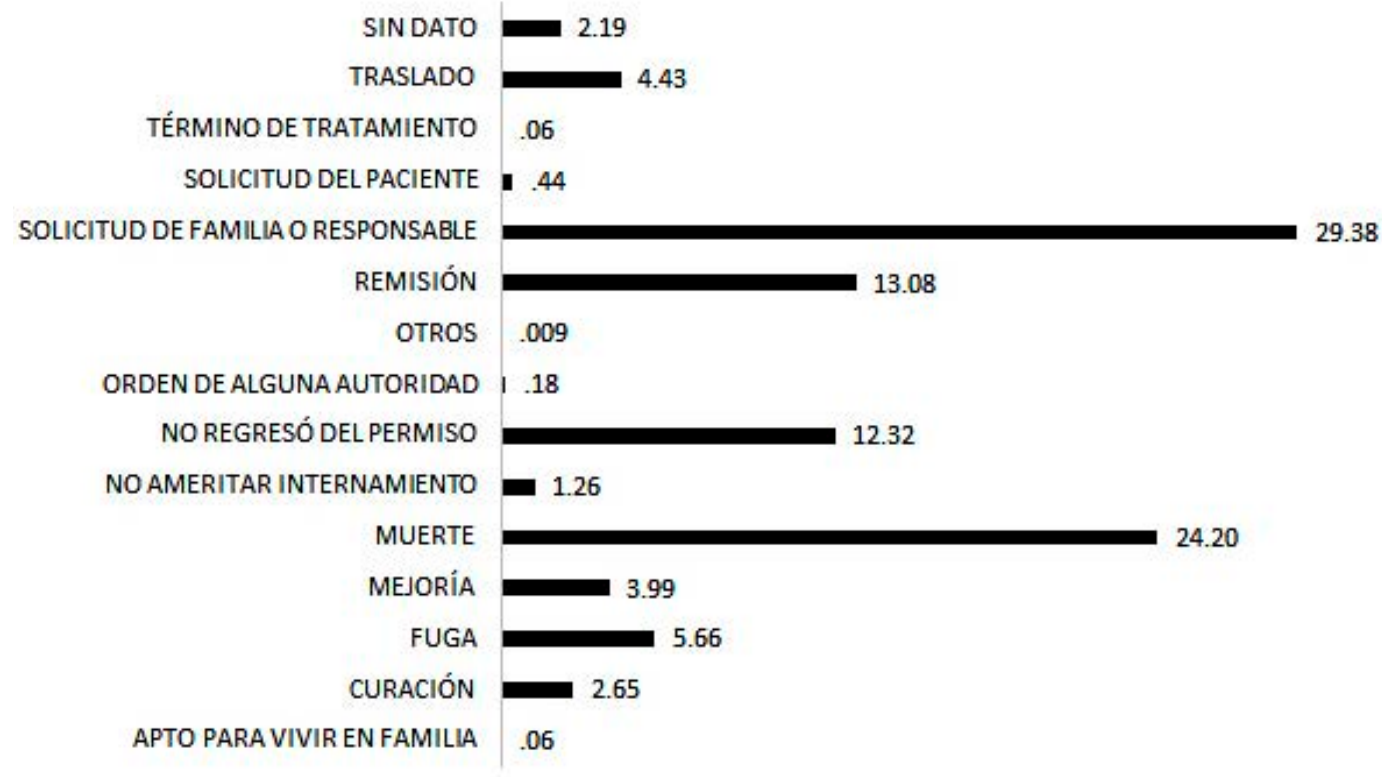

Fuente: BD-PAPIIT 
Tabla 6. Altas de pacientes agrupadas en categorías en ingresos y reingresos en el Manicomio La Castañeda, $1910-1968(n=10,641)$

\begin{tabular}{|l|c|}
\hline \multicolumn{1}{|c|}{ Categoría } & $\%$ \\
\hline Por indicación médica & 19,82 \\
\hline Por petición de la familia & 29,32 \\
\hline $\begin{array}{l}\text { Por intervención del } \\
\text { paciente }\end{array}$ & 18,29 \\
\hline Suma & $\mathbf{6 7 , 4 3}$ \\
\hline
\end{tabular}

Fuente: BD-PAPIIT

En la tabla 6 puede notarse que la familia jugó un papel muy preponderante, pero también el paciente que, ya fuera por fuga, por no regresar del permiso o por solicitar el alta, salía del encierro. Si comparamos las altas entre los pacientes que sólo ingresaron una vez y los que tuvieron reingresos (tabla 7), se aprecia que el primer ingreso de quienes después volverían a entrar a la institución rompe con las tendencias de todos los demás casos, ya que las altas fueron del 93.18\% frente al promedio de dos terceras partes ya mencionado. Estas altas obedecieron a la solicitud de la familia (41.25\%), no regresar del permiso (19.19\%), remisión (16.01\%) y fuga (8.39\%) (figura 3) aunadas a una bajísima mortalidad $(0,032 \%)$ en comparación con el promedio general del manicomio (24.20\%) y los promedios por tipo de ingreso (figuras 4,5 y 6 ). Si consideramos que el tiempo de estancia también fue muy atípico, como ya se dijo, se podría inferir que salir del manicomio de manera prematura pudo provocar el reingreso, pero esto es sólo una hipótesis que se tendría que verificar en cada caso con los expedientes clínicos. Lo que sí parece más claro es que quienes reingresaron tuvieron confinamientos extraordinariamente largos en relación con quienes sólo ingresaron una vez o con el primer ingreso de los que después volverían a la institución.

Estos hallazgos permiten concluir que las decisiones tomadas por la familia y los pacientes en relación con las altas contribuyeron al desahogo de la institución. Si bien no interpelan directamente los discursos de los médicos sobre la cronicidad, pues no podemos asegurar que hayan salido curados, sí nos llevan de nuevo a preguntarnos por el peso que los médicos concedieron a la sobrepoblación y a los crónicos en la vocación terapéutica del manicomio cuando las dos terceras partes de los internos salieron del manicomio. Avanzaremos ahora a otro nivel de análisis, el de los diagnósticos. En este caso, la muestra no proporciona los tratamientos dirigidos a cada tipo de diagnóstico, pero contamos con una investigación reciente que ha hecho una primera incursión sobre un tema con muchas aristas.

Figura 3. Condición de salida de pacientes que reingresan en el primer ingreso en el Manicomio La Castañeda, $1910-1968(n=2,167)$

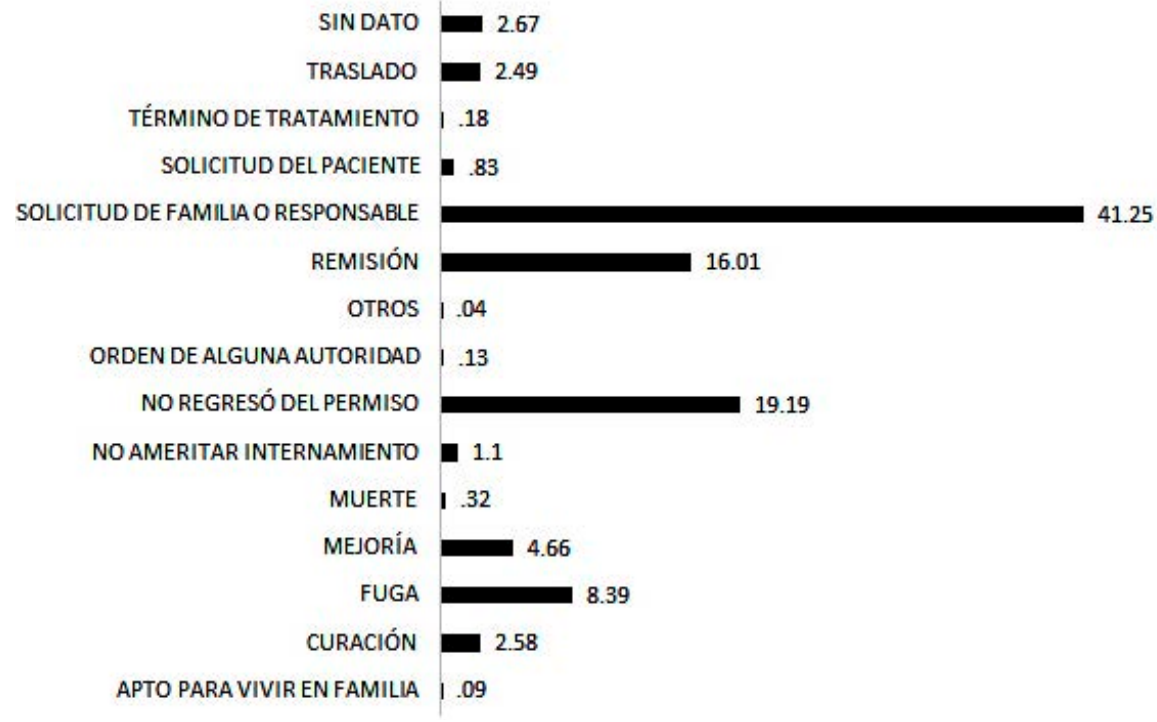

Fuente: BD-PAPIIT 
Figura 4. Condición de salida de pacientes con un solo ingreso en el Manicomio La Castañeda, 1910-1968 (n=8,474)

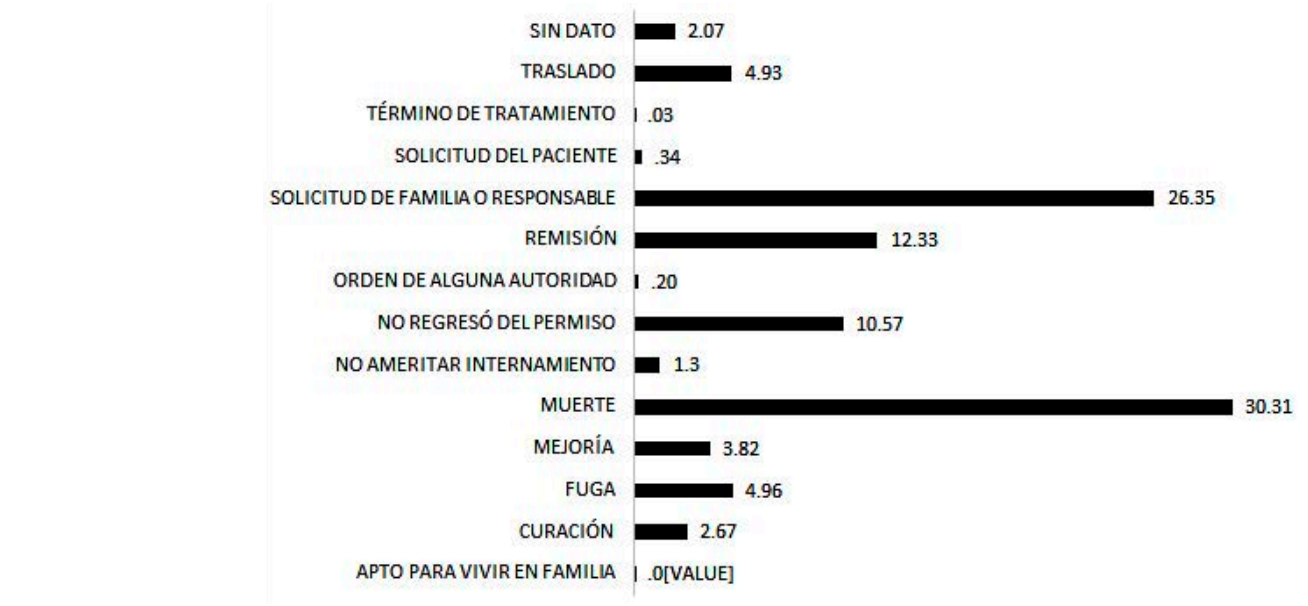

Fuente: BD-PAPIIT

Figura 5. Condición de salida de pacientes que reingresan en el segundo ingreso en el Manicomio La Castañeda, 1910-1968 $(n=1,419)$

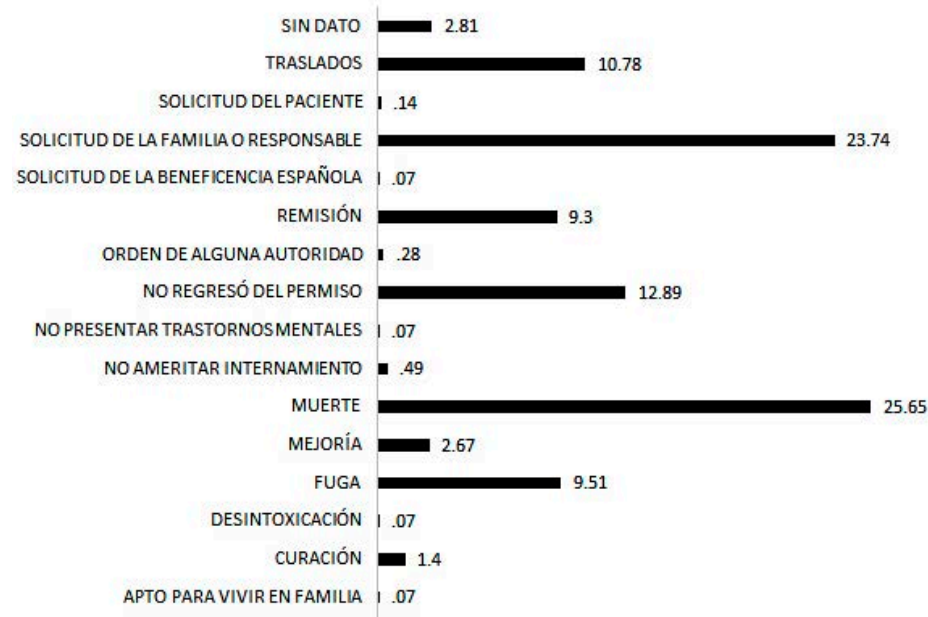

Fuente: BD-PAPIIT

Figura 6. Condición de salida de pacientes que reingresan en el tercer ingreso en el Manicomio La Castañeda, $1910-1968(n=441)$

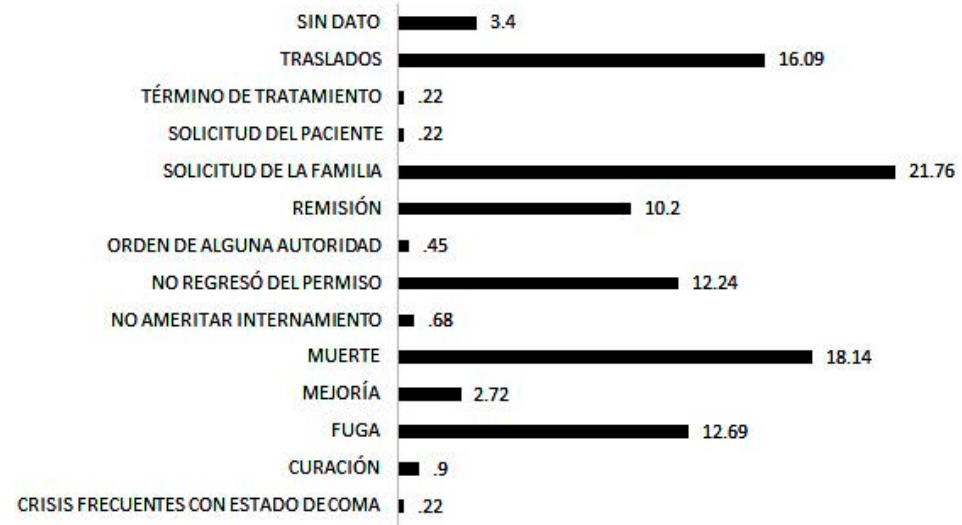

Fuente: BD-PAPIIT 
Tabla 7. Altas de pacientes agrupadas en categorías en ingresos y reingresos (primer, segundo y tercer ingreso) en el Manicomio La Castañeda, 1910-1968

\begin{tabular}{|l|c|c|c|c|}
\hline \multirow{2}{*}{ Categoría } & Ingreso único & Primer ingreso & Segundo ingreso & Tercer ingreso \\
\cline { 2 - 5 } & $(\mathrm{n}=8,474)$ & $(\mathrm{n}=2,167)$ & $(\mathrm{n}=1,419)$ & $(\mathrm{n}=441)$ \\
\cline { 2 - 5 } & $\%$ & $\%$ & $\%$ & $\%$ \\
\hline Por indicación médica & 18,9 & 23,52 & 13,46 & 14,05 \\
\hline Por petición de la familia & 26,29 & 41,25 & 23,74 & 21,31 \\
\hline Por intervención del paciente & 15,77 & 28,41 & 22,33 & 24,71 \\
\hline Suma & $\mathbf{6 0 , 9 6}$ & $\mathbf{9 3 , 1 8}$ & $\mathbf{5 9 , 5 3}$ & $\mathbf{6 0 , 0 7}$ \\
\hline
\end{tabular}

Fuente: BD-PAPIIT

Los criterios de clasificación de la nosología francesa, que habían sido utilizados en México desde el último tercio del siglo XIX, se fueron abandonando de manera progresiva en La Castañeda siendo sustituidos por el sistema clasificatorio del alemán Emil Kraepelin, lo que ocurrió hacia 1925, con la renovación generacional que dejó atrás a quienes se habían formado en el siglo anterior. Un nutrido grupo de jóvenes médicos se interesó por la investigación, la enseñanza y, en general, por la profesionalización de la psiquiatría y el fortalecimiento gremial mediante la publicación de revistas, la realización de congresos y la creación de asociaciones (Ríos Molina, 2016, pp. 17-39; Ríos Molina, et al., 2016, p. 4). Entre las novedades, adoptaron la manera kraepeliniana de concebir, clasificar y tratar las enfermedades mentales al clasificarlas según el curso de su evolución y no a partir del conjunto de síntomas que se presentaban en un momento dado, como se había hecho hasta entonces (Porter, 2003, pp. 175-176), por lo que su establecimiento significó sentar las bases de la psiquiatría moderna en México.

Siguiendo el sistema clasificatorio de Kraepelin, el diagnóstico con mayor representación lo tenemos en las "alteraciones mentales en intoxicaciones", concretamente alcoholismo y toxicomanía, que alcanzó el $23.2 \%$ del total de los diagnósticos de La Castañeda. Quienes fueron diagnosticados con estas patologías tuvieron en promedio una estancia muy breve en el manicomio en su primer ingreso, alrededor de 8 meses para los alcohólicos y de 2 meses y medio para los toxicómanos (Ríos Molina, et al., 2016, p. 9). Entre los alcohólicos la mortalidad fue más baja que en el resto de los pacientes del manicomio, $18 \%$ en promedio, y las altas reunidas bajo las categorías de indicación médica, petición de la familia e intervención del paciente anteriormente mencionadas, supe- raron el 77\% (Salazar, 2017). El segundo diagnóstico más importante lo constituyen los distintos tipos de esquizofrenias con el $19.9 \%$, cuyos pacientes desde su primer ingreso tuvieron estancias muy largas aunque éstas fueron disminuyendo de más de cuatro años entre 1910 y 1925 a menos de tres años entre 1926 y 1953, bajando a menos de un año entre 1954 y 1968, periodo en el que se introdujeron fármacos antipsicóticos (Ríos Molina, et al., 2016, pp. 11-13). El conjunto de las epilepsias representó la tercera enfermedad más diagnosticada con el 12.9\%; antes de 1930 se trató con relajantes del sistema nervioso como valeriana, bromuro de potasio y belladona, y a partir de esa fecha se incorporaron inyecciones de luminal, así como los barbitúricos Epamin, Hidontoina, Fenofarbital, Pentotal y Eskabarb con efectos de sedación y anticonvulsivos. Todo ello repercutió en una disminución del confinamiento manicomial que pasó de más de cuatro años antes de 1935 a menos de dos después de esa fecha (Maya, 2017). En cuarto lugar, tenemos la sífilis con el $9.4 \%$, padecimiento que presenta cuadros de demencia tras una evolución de diez o quince años tras contraer la enfermedad. Estos pacientes (militares y prostitutas sobre todo) acusaron una gran disminución a partir de 1950 con la introducción de la penicilina (Ríos Molina, et al., 2016, pp. 10-11); antes de esa década la mortalidad era muy alta, doblando a las altas por solicitud de la familia (Giraldo, 2017). Finalmente, el quinto grupo está representado por las oligofrenias con el 5.8\% del total y donde encontramos a la población de menor edad, ya que ingresaban desde la infancia (Ríos Molina, et al., 2016, pp. 6, 19). En este caso, la mortalidad es muy alta entre los nuevos ingresos, $47.2 \%$, y el tiempo de estancia también, más de cuatro años en promedio entre 1910 y 1949, disminuyendo a menos de dos años después de 1950, ya que en este 
grupo se encontraban los padecimientos con menor índice de curación y remisión (López Carrillo, 2017).

Con estos datos puede observarse que algunos padecimientos como el alcoholismo y las toxicomanías presentaron estadías muy breves, inferiores al promedio del manicomio, baja mortalidad y muchas altas, un respiro para los médicos ya que comprendían más del $20 \%$ de los padecimientos; el grupo de las epilepsias, las esquizofrenias y las enfermedades de origen sifilítico cursaron con estancias largas, pero desde los años 50 los anticomiciales, los antipsicóticos y la penicilina, aligeraron mucho la carga que estos padecimientos habían significado ya que, en conjunto, constituían el $42.9 \%$ de los casos diagnosticados; el grupo de las oligofrenias, se caracterizó por internaciones prolongadas, superiores al promedio de La Castañe$\mathrm{da}$, representando casi el $6 \%$ de los casos diagnosticados; otras enfermedades registraron estancias cortas como la histeria, pero con poco impacto por el bajo número de ingresos (Ríos Molina, et al., 2016, p. 6) o bien disminuyeron con el paso del tiempo como las enfermedades neurológicas que, tras registrar su pico más alto en el número de ingresos en 1949, fueron cayendo hasta que el Instituto Nacional de Neurología creado en 1964 absorbió la demanda (Vicencio, 2017).

\section{CONCLUSIONES}

En el manicomio La Castañeda, el discurso de los médicos que hemos analizado coloca la cronicidad de los pacientes y la incapacidad material de atender la demanda como las causas de la sobrepoblación, y a ésta como el factor que impidió cumplir con su misión científica: curar a los pacientes. La imagen que la asimila a un "almacén de enfermos mentales" no puede ser más elocuente. Los datos extraídos de los libros de admisión dan cuenta de la otra cara de la moneda: casi el $80 \%$ de los pacientes sólo tuvo un ingreso con una internación promedio de 15 meses, y las largas estancias de los que reingresaron no impactaron estadísticamente; la familia y los pacientes contribuyeron decididamente al desahogo de la institución ya que, junto con las altas de los médicos, las dos terceras partes de los enfermos salieron del manicomio, y desde los años cincuenta lo hicieron en el contexto de las nuevas terapéuticas farmacológicas. Ello nos acerca a la idea esbozada más arriba de que el manicomio pudo ser al mismo tiempo una institución médica y custodial. El último viaje realizado por los pacientes de La Castañeda, de camino a las nuevas instituciones donde serían trasladados ante el inminente cierre de la institución, también lo confirma.
Cuando en 1965 se tomó la decisión de clausurar y posteriormente demoler el manicomio, se encomendó a un comité de médicos rediagnosticar a todos los pacientes para establecer en qué tipo de institución se reubicarían. Las nuevas instalaciones serían de tres tipos: hospitales psiquiátricos para agudos, hospitales campestres para crónicos con pocas posibilidades de recuperación y hospitales-hogar para crónicos irrecuperables con estancias definitivas. Aunque no se tiene una cifra exacta, había en ese momento casi 3,000 pacientes. De ellos, menos de mil se consideraron agudos, ya que se pensó que unos 400 podían ser dados de alta con un seguimiento en consulta externa y unos 550, por ser de reciente evolución, deberían ser internados en hospitales para agudos; entre 1,000 y 1,300 fueron valorados como de muy larga evolución y habrían de ir a los hospitales campestres; alrededor de 500 calificados como crónicos irrecuperables y destinados a un hospital-hogar. Se dijo también que 200 niños serían llevados a centros pediátricos, sin especificar si eran agudos o crónicos. Eliminando a los niños, cuyo estado de cronicidad desconocemos, de los 2,750 pacientes que habrían de ser trasladados entre 1966 y 1968, 950 fueron considerados agudos y 1,800 crónicos (Calderón, 1995, p. 41). El hecho de que los agudos representaran una tercera parte de la población, nos lleva a pensar que fueron los responsables de la alta movilidad observada en la institución, de ahí que al momento del cierre constituyeran una minoría, mientras que los crónicos, 2 de cada 3, alimentaron el sentimiento de fracaso por dominar el escenario al ser mayoritarios. En este sentido, la permanencia de los crónicos con su deterioro progresivo debió impactar hasta el punto de desestimar los numerosos pacientes que dejaron la institución, y no precisamente en un ataúd. Por otro lado, el hecho de que las altas por curación fueran una minoría ya que, como vimos, muchos de los egresos estuvieron motivados por la intervención de la familia y de los propios pacientes, también debió contribuir a la frustración de los médicos que, inmersos en las difíciles condiciones de su trabajo, no alcanzaron a formarse una visión de conjunto, que esperamos haber logrado.

\section{AGRADECIMIENTOS}

Agradezco los comentarios a una primera versión de este trabajo por parte de Oliver Hernández, José Antonio Maya, Teresa Ordorika y Andrés Ríos Molina, así como las observaciones de los evaluadores anónimos. Las tablas y figuras que acompañan este artículo fueron elaboradas por Rosa Isela Flores Martínez, que mucho agradezco. 


\section{NOTAS}

1. Peón del Valle, 1937, p. 45.

2. La bibliografía es muy abundante. Puede verse, a manera de ejemplos, para Estados Unidos, (Grob, 1994, pp. 165-171; Shorter, 1997, pp. 33-48; Goodheart, 2010); para América Latina, (Ablard, 2003; Bassa, 2005; Sacristán, 2001); para España, (Campos, 2004; Villasante, 2005 y recientemente Plumed y Rojo Moreno, 2016). Este último sostiene que la idea de incurabilidad nunca hizo perder la confianza de los psiquiatras en su propia disciplina. Para el tratamiento moral y la selección que el propio Pinel hizo de las pacientes que consideraba curables, véase (Weiner, 2002, pp. 191-227).

3. Contamos con algunos trabajos basados en la metodología de la demografía psiquiátrica, aunque no se han propuesto analizar la cronicidad, sino el perfil general de los pacientes (sexo, edad, estado civil, ocupación, lugar de nacimiento, lugar de residencia, instancia remitente, diagnóstico, tiempo de internamiento, motivo del alta, traslados, fugas, mortalidad) con el fin de abordar otras problemáticas. Véase (Suzuki, 2003; Tierno, 2008; Goodheart, 2010; Ernst, 2012; Vázquez y Villasante, 2016).

4. La bibliografía sobre este manicomio es abundante. Puede verse para los primeros años, (Ríos Molina, 2009); una visión general en (Sacristán, 2010), y para el final de sus días, (Vicencio, 2014).

5. Contestación a la solicitud del agente del Ministerio Público sobre las listas de asilados para promover su interdicción, Archivo Histórico de la Secretaria de Salud (AHSS), Fondo Beneficencia Pública (F-BP), Sección Establecimientos Hospitalarios (S-EH), Serie Manicomio General (Se-MG), leg. 9, exp. 4, 1919.

6. Informe de actividades enviado por el director Manuel Guevara Oropesa al Jefe del Departamento Médico, AHSS, Fondo Manicomio General (F-MG), Sección Administrativa (S-A), leg. 34, exp. 4, 1943.

7. Actas de las sesiones del Consejo Psiquiátrico de Toxicomanías e Higiene Mental, AHSS, F-MG, S-A, leg. 48, exp. 2, actas 2, 4, 5 y 32, 1944-1945.

\section{BIBLIOGRAFÍA}

Ablard, Jonathan D. (2003), "The limits of psychiatric reform in Argentina, 1890-1946". En: Porter, Roy y Wright, David (eds.), The Confinement of the Insane. International Perspectives, 18001965, Cambridge, Cambridge University Press, pp. 226-247.

Álvarez, Raquel, Huertas, Rafael y Peset, José Luis (1993), “Enfermedad mental y sociedad en la Europa de la segunda mitad del siglo XIX", Asclepio, 45 (2), pp. 41-60.

Aragón, Enrique O. (1943), "La enajenación mental en México". En: Mis 31 años de académico (vol. I), México, Academia Nacional de Medicina de México, I, pp. 417-436.
8. La asistencia psiquiátrica en el Distrito Federal y en general el problema de la asistencia a alienados en la República, por Manuel Guevara Oropesa, AHSS, F-MG, S-A, leg. 43, exp. 4, 1943.

9. Proyecto de organización de un servicio social en el Manicomio General, por Mathilde Rodríguez Cabo, AHSS, Fondo Secretaría de Salubridad y Asistencia (F-SSA), Sección Subsecretaría de Salubridad y Asistencia (Se-SSA), leg. 3, exp. 14, 1944.

10. Relaciones de directores del Manicomio desde 1910 hasta 1962, AHSS, F-MG, S-A, leg. 57, exp. 11, 1962.

11. Cincuenta años de psiquiatría en el Manicomio General, por Jose Luis Patiño e Ignacio Sierra Macedo, AHSS, F-MG, S-A, leg. 56, exp. 6, 1960.

12. La base de datos se citará como BD-PAPIIT. Para más información sobre las variables que se recogieron, véase (Ríos Molina, et al., 2016). En todos los casos donde se presenta el resultado en promedios, se eliminaron los datos atípicos o outliers, con el fin de no distorsionar la media.

13. Algunos autores consideran que un caso es nuevo si cuando reingresa el paciente, ha transcurrido al menos un año fuera del manicomio, (Tierno, 2008, p. 99).

14. Reglamento del Manicomio General, AHSS, F-BP, S-EH, SeMG, leg. 16, exp. 9, 1913, art. 34.

15. Reglamento del Manicomio General, AHSS, F-BP, S-EH, SeMG, leg. 16, exp. 9, 1913, art. 38.

16. Reglamento del Manicomio General, AHSS, F-BP, S-EH, SeMG, leg. 16, exp. 9, 1913, art. 38.

17. La mortalidad representó el $24.20 \%$ y las restantes condiciones de salida (traslado a otra institución, no amerita internamiento, otros y sin dato), no alcanzan el $8 \%$.

18. Véase una entrevista periodística al director Guevara Oropesa en (Davó Lozano, 1941, pp. 39-41).

Bassa, Daniela (2005), “De la Pampa al Open Door. Terapias y tratamientos hacia los insanos en la primera mitad del siglo XX argentino". En: Di Liscia, María Silvia y Bohoslavsky, Ernesto (comps.) Instituciones y formas de control social en América Latina. 1840-1940. Una revisión, Buenos Aires, Universidad Nacional de General Sarmiento, Universidad Nacional de la Pampa, Prometeo Ediciones, pp. 115-139.

Calderón Narváez, Guillermo (1995), "Testimonio de trabajo en la Operación Castañeda”, Psiquis, 4 (3), pp. 40-45.

Campos Marín, Ricardo (2001), “De la higiene del aislamiento a la higiene de la libertad. La reforma de la institución manico- 
mial en Francia", Frenia. Revista de historia de la psiquiatría, I (1), pp. 37-64.

Campos Marín, Ricardo (2004), “La psiquiatría en la ciudad. Higiene mental y asistencia psiquiátrica extramanicomial en España en la década de 1920", Frenia. Revista de historia de la psiquiatría, IV (1), pp. 101-111.

Caponi, Sandra (2012), Loucos e degenerados. Uma genealogía da psiquiatría ampliada, Río de Janeiro, Editora FIOCRUZ.

Castel, Robert (1980), El orden psiquiátrico. La edad de oro del alienismo, Madrid, Las Ediciones de La Piqueta.

Comelles Esteban, Josep M. (1997), "Reforma asistencial y práctica económica. De la crisis del tratamiento moral a la hegemonía del kraepelinismo". En: Aparicio Basauri, Víctor (comp.), Orígenes y fundamentos de la psiquiatría en España, Madrid, Editorial Libro del Año, pp. 83-105.

Davó Lozano, Jorge (1941), “iYo estuve en el manicomio!”, Hoy, 14 de junio de 1941, pp. 39-41.

Dowbiggin, lan R. (1991), Inheriting Madness. Professionalization and Psychiatric Knowledge in Nineteenth Century France, Berkeley, California University Press.

Eraso, Yolanda (2002), “El trabajo desde la perspectiva psiquiátrica. Entre el tratamiento moral y el problema de la cronicidad en el manicomio de Oliva de Córdoba en las primeras décadas del siglo XX", Cuadernos de Historia, 5, pp. 33-63.

Ernst, Waltraud (2012), "The Limits of Comparison: Institutional Mortality Rates, Long-term Confinement and Causes of Death during the early Twentieth Century", History of Psychiatry, 23 (4), pp. 404-417.

Ernst, Waltraud (ed.) (2016), Work, Psychiatry and Society, c.1750-2015, Manchester University Press.

Giraldo Granada, Emmanuel Alejandro (2017), "Sífilis". En: Ríos Molina, Andrés (coord.), Los pacientes del Manicomio La Castañeda y sus diagnósticos. Una historia de la clínica psiquiátrica en México, 1910-1968, México, Instituto de Investigaciones Históricas de la UNAM, Instituto de Investigaciones Dr. José María Luis Mora.

Goodheart, Lawrence B. (2010), "From Cure to Custodianship of Insane Poor in Nineteenth-Century Connecticut", Journal of the History of Medicine and Allied Sciences, 65 (1), pp. 106-130.

Grob, Gerald N. (1994), The Mad Among Us. A History of the Care of America's Mentally III, Cambridge, Harvard University Press.

Hernández Lara, Oliver Gabriel (2014), La experiencia de atención psiquiátrica en México, de la forma asilar a la forma flexible. El caso del Instituto Nacional de Neurología y Neurocirugía, Tesis de doctorado en sociología, México, Benemérita Universidad Autónoma de Puebla.
Huertas García-Alejo, Rafael (1987), Locura y degeneración. Psiquiatría y sociedad en el positivismo francés, Madrid, Consejo Superior de Investigaciones Científicas.

Huertas, Rafael (2002), Organizar y persuadir. Estrategias profesionales y retóricas de legitimación de la medicina mental española (1875-1936), Madrid, Frenia.

Lantéri-Laura, Georges (1972), "La chronicité dans la psychiatrie française moderne. Note d'histoire théorique et sociaIe", Annales. Économies, Sociétés, Civilisations, XXVII (3), pp. 548-568.

López Carrillo, Ximena (2017), "Retraso mental”. En: Ríos Molina, Andrés (coord.), Los pacientes del Manicomio La Castañeda y sus diagnósticos. Una historia de la clínica psiquiátrica en México, 1910-1968, México, Instituto de Investigaciones Históricas de la UNAM, Instituto de Investigaciones Dr. José María Luis Mora.

Maya González, José Antonio (2017), “Epilepsia”. En: Ríos Molina, Andrés (coord.), Los pacientes del Manicomio La Castañeda y sus diagnósticos. Una historia de la clínica psiquiátrica en México, 1910-1968, México, Instituto de Investigaciones Históricas de la UNAM, Instituto de Investigaciones Dr. José María Luis Mora.

McGovern, Constance M. (1986), "The myths of social control and custodial oppression: patterns of psychiatric medicine in late nineteenth-century institutions", Journal of Social History, XX (1), pp. 3-23.

Mills, James H. (1999), "Reforming the Indian: Treatment Regimes in the Lunatic Asylums of British India, 1857-1880", The Indian Economic and Social History Review, 36 (4), pp. 407-429.

Peón del Valle, Juan (1937), "El culto de la curabilidad en psiquiatría", Revista de psiquiatría, neurología y medicina legal, 4 (21), p. 45.

Plumed Domingo, José Javier y Rojo Moreno, Luis Miguel (2016), "El tratamiento de la locura entre los siglos XIX y XX: los discursos sobre la cura en la medicina mental española, 1890-1917", História, Ciências, Saúde, Manguinhos, 23 (4), pp. 985-1002.

Porter, Roy (2003), Breve historia de la locura, Madrid, Turner, Fondo de Cultura Económica.

Ríos Molina, Andrés (2009), La locura durante la Revolución Mexicana. Los primeros años del Manicomio La Castañeda, 1910-1920, México, El Colegio de México.

Ríos Molina, Andrés (2016), Cómo prevenir la locura. Psiquiatría e higiene mental en México, 1934-1950, México, Instituto de Investigaciones Históricas de la Universidad Nacional Autónoma de México/Siglo XXI Editores.

Ríos Molina, Andrés (coord.) (2017), Los pacientes del Manicomio La Castañeda y sus diagnósticos. Una historia de la 
clínica psiquiátrica en México, 1910-1968, México, Instituto de Investigaciones Históricas de la UNAM, Instituto de Investigaciones Dr. José María Luis Mora.

Ríos Molina, Andrés, Sacristán, Cristina, Ordorika Sacristán, Teresa y López Carrillo, Ximena (2016), "Los pacientes del Manicomio La Castañeda y sus diagnósticos. Una propuesta desde la historia cuantitativa (México, 1910-1968)", Asclepio 68 (1): p136, http://dx.doi.org/10.3989/asclepio.2016.15

Sacristán, Cristina (2001), “Una valoración sobre el fracaso del manicomio de La Castañeda como institución terapéutica, 1910-1944", Secuencia. Revista de historia y ciencias sociales, 51, pp. 91-120.

Sacristán, Cristina (2005), “Por el bien de la economía nacional. Trabajo terapéutico y asistencia pública en el Manicomio de La Castañeda de la ciudad de México, 1929-1932", História, Ciências, Saúde, Manguinhos, 12 (3), pp. 675-692.

Sacristán, Cristina (2010), “La contribución de La Castañeda a la profesionalización de la psiquiatría Mexicana", Salud Mental. Revista del Instituto Nacional de Psiquiatría, 33 (6), pp. 473-480.

Sacristán, Cristina (2011), “Un Estado sin memoria. La abolición ideológica de la institución manicomial en México, 19451968", VERTEX. Revista argentina de psiquiatría, XXII (98), pp. 314-317.

Sacristán, Cristina (2017), "La clínica psiquiátrica en el Pabellón Central". En: Ríos Molina, Andrés (coord.), Los pacientes del Manicomio La Castañeda y sus diagnósticos. Una historia de la clínica psiquiátrica en México, 1910-1968, México, Instituto de Investigaciones Históricas de la UNAM, Instituto de Investigaciones Dr. José María Luis Mora.

Salazar Bermúdez, Alejandro (2017), “Alcoholismo". En: Ríos Molina, Andrés (coord.), Los pacientes del Manicomio La Castañeda y sus diagnósticos. Una historia de la clínica psiquiátrica en México, 1910-1968, México, Instituto de Investigaciones Históricas de la UNAM, Instituto de Investigaciones Dr. José María Luis Mora.

Secretaría de Salubridad y Asistencia (1964), “Dirección General de Neurología, Salud Mental y Rehabilitación". En: Memoria 1958-1954, México, Secretaría de Salubridad y Asistencia, pp. 150-156.

Shorter, Edward (1997), A History of Psychiatry. From the Era of the Asylum to the Age of Prozac, New York, John Wiley \& Sons, Inc.
Suzuki, Akihito (2003), "A brain hospital in Tokyo and its private and public patients, 1926-1945", History of Psychiatry, 14 (3), pp. 337-360.

Tierno, Raquel (2008), “Demografía psiquiátrica y movimientos de la población del Manicomio Nacional de Santa Isabel (1931-1952)", Frenia. Revista de historia de la psiquiatría, VIII, pp. 97-130.

Valdés Fernández, Marisa (1995), La salud mental en el Porfiriato. La construcción de "La Castañeda", Tesis de licenciatura en historia, México, Universidad Nacional Autónoma de México.

Valverde Pérez, Nuria (2016), "Meanings of Waves: Electroencephalography and Society in Mexico City, 1940-1950", Science in Context, 29 (4), pp. 451-472, http://dx.doi. org/10.1017/S0269889716000223

Vázquez de la Torre, Paloma y Villasante, Olga (2016), "Psychiatric care at a National Mental institution during the Spanish Civil War (1936-39): Santa Isabel de Leganés", History of Psychiatry, 27 (1), pp. 1-14.

Velasco Suárez, Manuel (1964), "Dirección General de Neurología, Salud Mental y Rehabilitación", Salud Pública de México, época V, VI (6), pp. 1187-1189.

Vezzetti, Hugo (1985), La locura en la Argentina, Buenos Aires, Paidós.

Vicencio Muñoz, Daniel (2014), La Operación Castañeda. Una reforma asistencial de la psiquiatría en México, 1940-1968, tesis de maestría, México, Universidad Nacional Autónoma de México.

Vicencio Muñoz, Daniel (2017), "Trastornos neurológicos". En: Ríos Molina, Andrés (coord.), Los pacientes del Manicomio La Castañeda y sus diagnósticos. Una historia de la clínica psiquiátrica en México, 1910-1968, México, Instituto de Investigaciones Históricas de la UNAM, Instituto de Investigaciones Dr. José María Luis Mora.

Villasante, Olga (2005), "Las instituciones psiquiátricas madrileñas en el periodo de entresiglos: asistencia pública frente a sanatorios privados", Frenia. Revista de historia de la psiquiatría, V (1), pp. 69-99.

Weiner, Dora (2002), Comprender y curar. Philippe Pinel (17451826). La medicina de la mente, México, Fondo de Cultura Económica. 